\title{
Expression and secretion of neuregulin-1 in cardiac microvascular endothelial cells treated with angiogenic factors
}

\author{
CHENGQIANG WU ${ }^{1}$, CHUN GUI $^{1}$, LANG LI $^{1}$, YIHENG PANG $^{2}$, ZHONGLI TANG $^{3}$ and JING WEI ${ }^{1}$ \\ ${ }^{1}$ Department of Cardiology, The First Affiliated Hospital of Guangxi Medical University; \\ ${ }^{2}$ Department of Cardiology, The Second Affiliated Hospital of Guangxi Medical University, Nanning, Guangxi 530021; \\ ${ }^{3}$ Department of Cardiology, Daoxian People's Hospital, Yongzhou, Hunan 425300, P.R. China
}

Received October 12, 2017; Accepted January 19, 2018

DOI: $10.3892 / \mathrm{etm} .2018 .5811$

\begin{abstract}
Neuregulin-1 (NRG-1) is a positive regulator of angiogenesis, which suggests there may be an association between NRG-1 and angiogenic factors. The aim of the present study was to investigate the effect of treating human cardiac microvascular endothelial cells (HCMECs) with angiogenic factors on NRG-1 expression and secretion. HCMECs were cultured and stimulated with vascular endothelial growth factor (VEGF; $100 \mathrm{ng} / \mathrm{ml}$ ), angiopoietin (Ang)-1 (100 ng/ml) or Ang-2 (100 ng/ml) under normal or hypoxia/serum deprivation (Hypo/SD) conditions for $24 \mathrm{~h}$. The expression of ErbB receptors and NRG-1 in HCMECs was measured by western blot analysis and the secretion of NRG-1 in HCMECs was determined by ELISA. The results demonstrated that ErbB2, ErbB3 and ErbB4 were expressed in HCMECs and that ErbB2 expression levels were notably higher than those of ErbB3 and ErbB4. Under normal culture conditions the expression and secretion of NRG-1 was significantly increased in HCMECs treated with VEGF or Ang-1 $(\mathrm{P}<0.05)$, however levels significantly decreased in HCMECs treated with Ang-2 $(\mathrm{P}<0.05)$. Under Hypo/SD conditions the expression and secretion of NRG-1 significantly increased $(\mathrm{P}<0.05)$ and VEGF or Ang-1 treatment significantly increased these effects further $(\mathrm{P}<0.05)$. Conversely Ang-2 treatment significantly decreased these effects $(\mathrm{P}<0.05)$. The expression and release of NRG-1 were significantly increased in HCMECs with VEGF or Ang-1 treatment $(\mathrm{P}<0.05)$, which suggests that VEGF and Ang-1 may regulate myocardial angiogenesis and survival via the NRG-1/ErbB signaling pathway.
\end{abstract}

Correspondence to: Dr Chun Gui, Department of Cardiology, The First Affiliated Hospital of Guangxi Medical University, 22 Shuangyong Road, Nanning, Guangxi 530021, P.R. China E-mail: gui_chun@126.com

Key words: neuregulin-1, ErbB receptors, vascular endothelial growth factor, angiopoietins

\section{Introduction}

Neuregulin-1 (NRG-1) is a member of the epidermal growth factor family. Its receptors are the ErbB family of tyrosine kinase transmembrane receptors, including the ErbB2, ErbB3 and ErbB4. In the heart, NRG-1 is synthesized and released by the endocardial and cardiac microvascular endothelium (1). NRG-1 is essential for the development of the cardiovascular system and the maintenance of adult heart function $(2,3)$. Recently, growing evidence indicates that NRG-1 is a positive regulator of angiogenesis $(4,5)$. Russell et al reported that NRG-1 and ErbB receptors are expressed in vascular endothelial cells, and NRG-1 treatment can induce angiogenesis of endothelial cells in vitro (6). Hedhli et al reported that NRG-1 of endothelial production is necessary for angiogenesis and arteriogenesis induced by femoral artery ligation, and exogenous administration of NRG-1 can enhance this process (7). Xiao et al reported elevated expression of NRG-1 can increase the number of micro-vessels formed in the ischemic myocardium (8).

Angiogenesis is a highly regulated process requiring coordinated signaling events among a variety of angiogenic factors. Vascular endothelial growth factor (VEGF), angiopoietin-1 (Ang-1) and angiopoietin-2 (Ang-2) play essential roles in angiogenesis. Both Flk1 and Tie-2 receptors are exclusively expressed in endothelial cells. The VEGF/Flk1 signal system takes the lead in new vessel formation, and Ang-1/Tie-2 signal system plays a critical role in vascular maturation and stabilization. Notably Ang-2 represents a natural Ang1/Tie2 inhibitor, may cause destabilization and initiate neovascularization (9).

Our previous study demonstrated that serum NRG-1 $\beta$ levels are positively correlated with serum VEGF and Ang-1 levels in patients with diabetes or unstable angina pectoris (10). It has been reported that NRG-1 stimulation can increase the expression and secretion of VEGF in tumor cells (11) and endothelial cells (12). Nakaoka et al reported that NRG-1 stimulation can increase mRNA expression of Ang-1 in the heart (13). There may be a relationship between NRG-1 and these angiogenic factors. However, it is not clear whether stimulation with these angiogenic factors can increase NRG-1 production in endothelial cells. With this possibility in mind, the aim of this study was to investigate the effects of angiogenic factors 
treatment on the expression and secretion of NRG-1 in human cardiac microvascular endothelial cells (HCMECs) under normal or hypoxia/serum deprivation (Hypo/SD) culture conditions.

\section{Materials and methods}

Cells and reagents. HCMECs were obtained from ScienCell Research Laboratories (Carlsbad, CA, USA); anti-ErbB2 rabbit monoclonal antibody, anti-ErbB3 rabbit monoclonal antibody and anti-ErbB4 rabbit monoclonal antibody were obtained from Cell Signaling Technology, Inc. (Danvers, MA, USA); anti-NRG-1 rabbit polyclonal antibody was obtained from R\&D Systems (Minneapolis, MN, USA); anti-GAPDH rabbit polyclonal antibody was obtained from Signalway Antibody LLC (College Park, MD, USA); VEGF and Ang-1 were obtained from ProSpec-Tany TechnoGene Ltd. (Nessziona, Israel); Ang-2 was obtained from Peprotech (Oak Park, CA, USA); NRG-1 $\beta$ ELISA kit was obtained from RayBiotech (Norcoss, GA, USA). Myocardial tissues of rat were obtained from our laboratory.

HCMECs culture and grouping. Primary HCMECs from liquid nitrogen were completely thawed in a $37^{\circ} \mathrm{C}$ water bath. The cells were cultured in $25 \mathrm{~cm}^{2}$ culture flasks with endothelial cell medium (ScienCell Research Laboratories) supplemented with $10 \%$ fetal bovine serum (FBS) in a $37^{\circ} \mathrm{C}$ and $5 \% \mathrm{CO}_{2}$ incubator. Medium was changed every 3 days. At confluence the cells were passaged. In all experiments, cells from the 4 th passage were used. According to the need of tests, cells were stimulated with VEGF (100 ng/ml), Ang-1 (100 ng/ml) or Ang-2 (100 ng/ml) under normal or Hypo/SD conditions for $24 \mathrm{~h}$. Hypo/SD represents both of components in ischemia in vivo. In our model of Hypo/SD, cells were incubated in a modular incubator chamber and infused with mixed gas $\left(95 \% \mathrm{~N}_{2}\right.$ and $\left.5 \% \mathrm{CO}_{2}\right)$ at $37^{\circ} \mathrm{C}$ and $\mathrm{O}_{2}<0.5 \%$. Under normal culture condition, experimental cells were divided into four groups, including control group, VEGF treatment group, Ang-1 treatment group and Ang-2 treatment group. Under Hypo/SD condition, experimental cells were divided into five groups, including control group, Hypo/SD group, Hypo/SD+VEGF treatment group, Hypo/SD+Ang-1 treatment group and Hypo/SD+Ang-2 treatment group.

Western blot analysis. Cells were harvested into $1.5 \mathrm{ml}$ micro-tubes, RIPA buffer with PMSF $(0.1 \mathrm{mM})$ was added immediately, then protein extraction and quantification were performed. Total protein was fractionated by SDS gel electrophoresis and transferred to a visualization membrane. The membrane was blocked with $5 \%$ nonfat milk for $1 \mathrm{~h}$ at room temperature followed by overnight incubation at $4^{\circ} \mathrm{C}$ with primary antibodies. Primary antibody binding was detected using a horseradish peroxidase conjugated secondary antibody and an enhanced chemiluminescence detection system (Thermo Fisher Scientific, Inc., Waltham, MA, USA). Membrane bands were analyzed using Image-analysis software (Quantity One; Bio-Rad Laboratories, Inc., Hercules, CA, USA).

ELISA analysis. The same quantity of HCMECs were plated in each $25 \mathrm{~cm}^{2}$ culture flask and cultured in an incubator as previously described. After $48 \mathrm{~h}$, the culture medium (the same volume each flask) was changed. Under normal or Hypo/SD culture conditions, cells were cultured with VEGF, Ang-1 or Ang-2 treatment for $24 \mathrm{~h}$. This conditioned medium was then collected and concentrated using 3kD Amicon Ultra-15 Centrifugal Filter Units (EMD Millipore, Billerica, MA, USA). The remaining concentrated medium $(250 \mu \mathrm{l})$ was transferred to another tube and stored at $-80^{\circ} \mathrm{C}$ until use. $\mathrm{NRG}-1 \beta$ secreted in the HCMECs culture medium was detected by a commercially available ELISA kit according to the manufacturer's protocol. Standards or samples $(100 \mu 1)$, detection reagent, substrate solution and stop solution were sequentially pipette into wells and incubated with repeated washing. The reaction product was analyzed spectrophotometrically at $450 \mathrm{~nm}$ with a plate reader, and sample values were calculated using a standard curve.

Statistical analysis. Numerical values are expressed as the mean \pm standard deviation. The data were analyzed using the SPSS 16.0 statistic software package (SPSS, Inc., Chicago, IL, USA). One-way analysis of variance was performed, and post hoc multiple comparisons were conducted with S-N-K. $\mathrm{P}<0.05$ was considered to indicate a statistically significant difference.

\section{Results}

ErbB receptors were expressed in HCMECs. It is well established that NRG-1 is expressed in HCMECs, but there are no studies reporting whether ErbB receptors are expressed in HCMECs. To this end, we screened for the expression of ErbB2, ErbB3 and ErbB4 receptors in HCMECs using western blot. Since ErbB receptors are expressed in myocardial tissues, so myocardial tissues of rat were used as a positive contrast. Western blot results showed all three ErbB receptors were expressed in HCMECs, and ErbB2 expression levels were much higher than ErbB3 and ErbB4 expression levels. However, ErbB4 expression levels were much higher than ErbB2 and ErbB3 expression levels in myocardial tissues (Fig. 1).

NRG-1 expression was regulated by angiogenic factors. We examined the effects of VEGF, Ang-1 and Ang-2 treatment on NRG-1 expression in HCMECs under normal or Hypo/SD conditions. Under normal culture condition, NRG-1 expression was significantly increased in the VEGF treatment group and Ang-1 treatment group, but significantly decreased in Ang-2 treatment group as compared with that of the control group ( $\mathrm{P}<0.05$; Fig. 2A). Under Hypo/SD condition, Hypo/SD stimulation significantly increased NRG-1 expression, and VEGF or Ang-1 treatment significantly further increased NRG-1 expression, but Ang-2 treatment significantly decreased NRG-1 expression $(\mathrm{P}<0.05$; Fig. 2B).

$N R G-1$ secretion was regulated by angiogenic factors. We further examined the effects of VEGF, Ang-1 and Ang-2 treatment on NRG-1 $\beta$ secretion in HCMECs under normal or Hypo/SD conditions. Under normal culture condition, the results demonstrated that VEGF or Ang-1 treatment significantly increased NRG-1 $\beta$ secretion, but Ang-2 treatment significantly decreased NRG-1 $\beta$ secretion $(\mathrm{P}<0.05$; 
A HCMECS

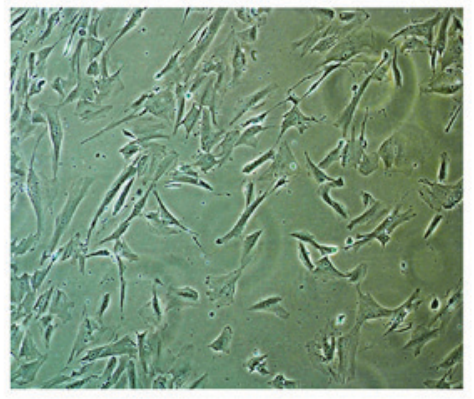

B HCMECS MC

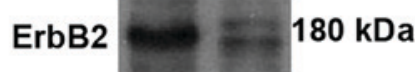

ErbB3 $185 \mathrm{kDa}$

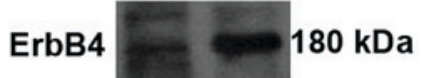

GAPDH $36 \mathrm{kDa}$

Figure 1. ErbB receptors were expressed in HCMECs. (A) The shape of the 4th passages HCMECs under a phase contrast microscope (magnification, x100). (B) ErbB2, ErbB3 and ErbB4 were expressed in HCMECs, MCs were used as a positive contrast. HCMECs, human cardiac microvascular endothelial cells; $\mathrm{MC}$, rat myocardial tissue.
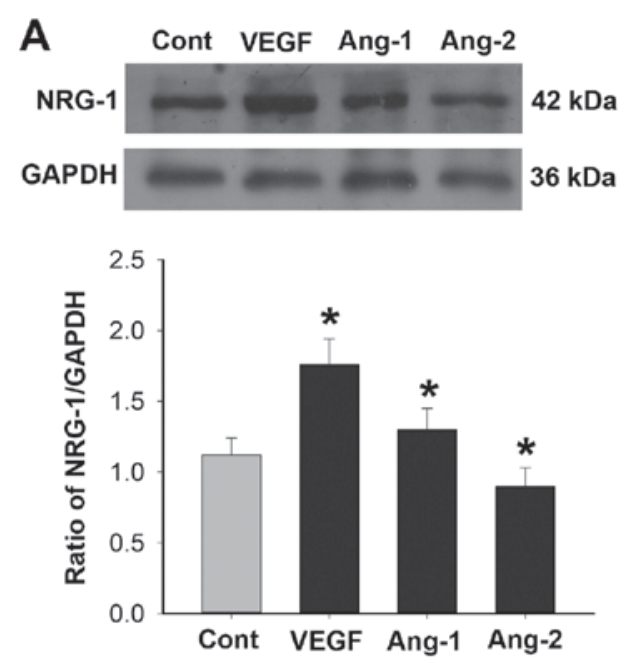
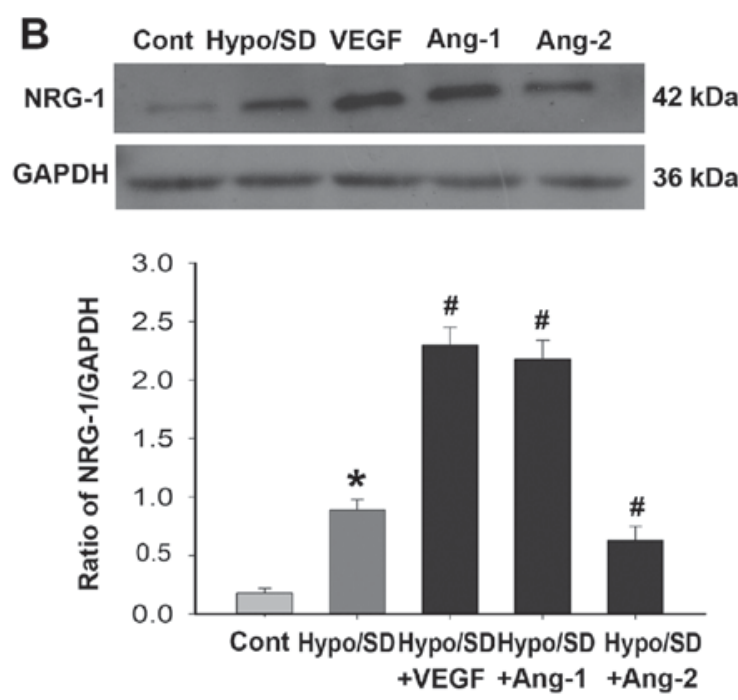

Figure 2. NRG-1 protein expression in HCMECs in different groups. (A) NRG-1 protein expression in HCMECs with angiogenic factors stimulation under normal conditions. (B) NRG-1 protein expression in HCMECs with angiogenic factors stimulation under Hypo/SD conditions. "P<0.05 vs. the control group; ${ }^{\text {"P}} \mathrm{P}<0.05$ vs. the Hypo/SD only group. HCMECs, human cardiac microvascular endothelial cells; NRG-1, neuregulin-1; Hypo/SD, hypoxia/serum deprivation.
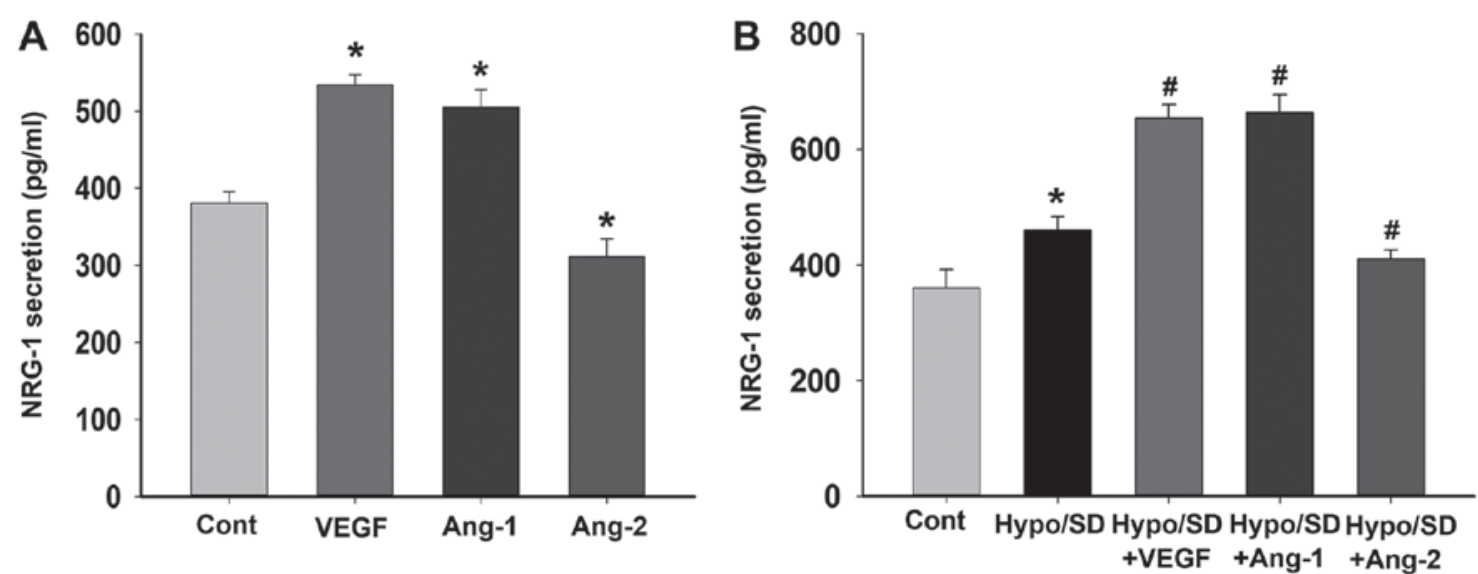

Figure 3. NRG-1 $\beta$ secretion in HCMECs in different groups. (A) NRG-1 $\beta$ secretion in HCMECs with angiogenic factors stimulation under normal conditions. (B) NRG-1 $\beta$ secretion in HCMECs with angiogenic factors stimulation under Hypo/SD conditions. ${ }^{*} \mathrm{P}<0.05$ vs. the control group; ${ }^{\#} \mathrm{P}<0.05$ vs. the Hypo/SD only group. HCMECs, human cardiac microvascular endothelial cells; NRG-1 $\beta$, neuregulin-1 $\beta$; Hypo/SD, hypoxia/serum deprivation.

Fig. 3A). Under Hypo/SD condition, the results demonstrated that Hypo/SD stimulation significantly increased NRG-1 $\beta$ secretion, and VEGF or Ang-1 treatment significantly further increased NRG-1 $\beta$ secretion, but Ang-2 treatment significantly decreased NRG-1 $\beta$ secretion $(\mathrm{P}<0.05$; Fig. 3B). 


\section{Discussion}

This study reported, for the first time, the effects of angiogenic factors treatment on the expression and secretion of NRG-1 in HCMECs. Results demonstrated that ErbB2, ErbB3 and ErbB4 receptors were expressed in HCMECs, and the expression and secretion of NRG-1 in HCMECs were upregulated by Hypo/SD, VEGF or Ang-1 treatment, but were downregulated by Ang-2 treatment.

HCMECs can directly interact with adjacent cardiomyocytes and are the main cells-type involved in angiogenesis. Through autocrine and paracrine regulation, HCMECs can increase cardiac angiogenesis and of myocyte survival. Growth factors, such as Angs, VEGF and NRG-1, are involved in these interactions. In the heart, NRG-1 is synthesized and released by the endocardial and cardiac microvascular endothelium $(1,2)$. Hedhli et al have reported that NRG-1 plays an important role in cardiac myocyte protection and angiogenic responses to ischemia injury, and NRG-1 secretion is significantly increased in cardiac endothelial cells in response to hypoxia (14). Our study also showed the expression and secretion of NRG-1 were increased in HCMECs with Hypo/SD stimulation. Hypo/SD mimics the environments of ischemia in vivo. This result is also consistent with the findings of previous in vivo studies. Kuramochi et al reported that NRG-1 released from the endothelium is acutely promoted in models of ischemia/reperfusion (15). Ky et al reported that circulating NRG-1 levels are increased in chronic heart failure of ischemic but not of non-ischemic etiology (16). Geisberg et al reported plasma NRG-1 $\beta$ levels were statistically higher in patients with stress-induced ischemia, and NRG-1 $\beta$ levels were inversely correlated with ischemia severity (17). These findings suggest that ischemia may be an important trigger for endothelial NRG-1 synthesis and release.

Our results further illustrate that VEGF or Ang-1 treatment can increase the expression and secretion of NRG-1 in HCMECs, but Ang-2 treatment has opposite effect. Regulated cleavage and release of transmembrane growth factors have been recognized as a common and important mechanism for autocrine and paracrine signaling. Kalinowski et al reported that pro-forms of NRG-1 can be cleaved by TNF converting enzyme in response to the inflammatory cytokines IL- 6 and IFN (18). Lemmens et al reported that mechanical strain increases endothelial NRG-1 synthesis and release, but angiotensin II and adrenergic agonists decrease endothelial NRG-1 synthesis and release $(1,19)$.

The mechanisms of the inverse relationship of Ang-2 on NRG-1 are not clear. Although Ang-1 and Ang-2 share similar binding affinities for the Tie 2 receptor, they have opposing effects on receptor activation. Ang-1 induces receptor phosphorylation and contributes to blood vessel stabilization. The roles of Ang-2 in angiogenesis are complicated, depending on the availability of VEGF. In general, Ang-2 antagonizes the actions of Ang-1 and is associated with blood vessel growth or regression (20). Previous studies have reported that NRG-1 stimulation can increase the expression of VEGF and Ang-1 in different kinds of cells (10-12). This is the first report regarding the effect of angiogenic factors treatment on the expression and secretion of NRG-1, but the limitation of this study is that we only investigated the effect of an angiogenic factor alone.
These results suggest VEGF and Ang-1 may increase myocardial angiogenesis and survival via NRG-1/ErbB signaling.

Russell et al demonstrated that ErbB2, ErbB3 and ErbB4 are expressed in human umbilical vein endothelial cells and the ErbB2 expression levels are the highest among three ErbB receptors (6). Lok et al reported ErbB2 and ErbB3 receptors are expressed in brain endothelial cells, but ErbB4 was not detected (21). Zhao et al reported that ErbB4 expression is quite prominent in cardiac myocytes, while ErbB2 is only barely detectable and ErbB3 is not detectable (22). This study reported, for the first time, that three ErbB receptors are expressed in HCMECs, and ErbB2 expression levels are higher than the expression levels of ErbB3 and ErbB4. However, ErbB4 expression levels were much higher than ErbB2 and ErbB3 expression levels in myocardial tissues. This results are consistent with previous studies.

NRG-1 binds with high affinity only to ErbB3 and ErbB4, but can very efficiently activate ErbB2 as a heterodimer with either receptor. Hedhli et al found three ErbB receptors are expressed in human coronary artery smooth muscle cells (SMC) (4), and this study found HCMECs express both NRG-1 and ErbB receptors. So these findings indicate HCMECs and SMC can participate in both autocrine and paracrine regulation via NRG-1/ErbB signaling, an important potential mechanism for regulation of vascular responses. ErbB2 and ErbB4 are expressed in cardiomyocytes, the crosstalk between endothelial cells and cardiomyocytes via this pathway can have profound effects on myocardial function and survival (23).

In conclusion, the expression and release of NRG-1 are increased in HCMECs in the presence of VEGF or Ang-1, demonstrating that VEGF and Ang-1 may regulate myocardial angiogenesis and survival via NRG-1/ErbB signaling.

\section{Acknowledgements}

This study was supported by the National Natural Science Foundation of China (grant no. 81460063) and Guangxi Natural Science Foundation (grant no. 2014GXNSFDA118024).

\section{References}

1. Lemmens K, Segers VF, Demolder M and De Keulenaer GW: Role of neuregulin-1/ErbB2 signaling in endothelium-cardiomyocyte crosstalk. J Biol Chem 281: 19469-19477, 2006.

2. Odiete O, Hill MF and Sawyer DB: Neuregulin in cardiovascular development and disease. Circ Res 111: 1376-1385, 2012.

3. Rupert CE and Coulombe KL: The roles of neuregulin-1 in cardiac development, homeostasis, and disease. Biomark Insights 10 (Suppl 1): S1-S9, 2015.

4. Hedhli N, Kalinowski A and S Russell K: Cardiovascular effects of neuregulin-1/ErbB signaling: Role in vascular signaling and angiogenesis. Curr Pharm Des 20: 4899-4905, 2014.

5. Hedhli N and Russell KS: Cytostatic drugs, neuregulin activation of erbB receptors and angiogenesis. Curr Hypertens Rep 12: 411-417, 2010.

6. Russell KS, Stem DF, Polverini PJ and Bender JR: Neuregulin activation of ErbB receptors in vascular endothelium leads to angiogenesis. Am J Physiol 277: H2205-H2211, 1999.

7. Hedhli N, Dobrucki LW, Kalinowski A, Zhuang ZW, Wu X, Russell RR III, Sinusas AJ and Russell KS: Endothelial-derived neuregulin is an important mediator of ischaemia-induced angiogenesis and arteriogenesis. Cardiovasc Res 93: 516-524, 2012.

8. Xiao J, Li B, Zheng Z, Wang M, Peng J, Li Y and Li Z: Therapeutic effects of neuregulin-1 gene transduction in rats with myocardial infarction. Coron Artery Dis 23: 460-468, 2012. 
9. Karamysheva AF: Mechanisms of angiogenesis. Biochemistry (Mosc) 73: 751-760, 2008 .

10. Zeng Z, Gui C, Nong Q, Du F and Zhu L: Serum neuregulin-1 $\beta$ levels are positively correlated with VEGF and Angiopoietin-1 levels in patients with diabetes and unstable angina pectoris. Int J Cardiol 168: 3077-3079, 2013.

11. Yonezawa M, Wada K, Tatsuguchi A, Akamatsu T, Gudis K, Seo T, Mitsui K, Nagata K, Tanaka S, Fujimori S and Sakamoto C: Heregulin-induced VEGF expression via the ErbB3 signaling pathway in colon cancer. Digestion 80: 215-225, 2009.

12. Iivanainen E, Paatero I, Heikkinen SM, Junttila TT, Cao R, Kint P, Jaakkola PM, Cao Y and Elenius K: Intra- and extracellular signaling by endothelial neuregulin-1. Exp Cell Res 313: 2896-2909, 2007.

13. Nakaoka Y, Nishida K, Narimatsu M, Kamiya A, Minami T, Sawa H, Okawa K, Fujio Y, Koyama T, Maeda M, et al: Gab family proteins are essential for postnatal maintenance of cardiac function via neuregulin-1/ErbB signaling. J Clin Invest 117: 1771-1781, 2007.

14. Hedhli N, Huang Q, Kalinowski A, Palmeri M, Hu X, Russell RR and Russell KS: Endothlium-derived neuregulin protects the heart against ischemic injury. Circulation 123: 2254-2262, 2011.

15. Kuramochi Y, Cote GM, Guo X, Lebrasseur NK, Cui L, Liao R and Sawyer DB: Cardiac endothelial cells regulate reactive oxygen species-induced cardiomyocyte apoptosis through neuregulin-1beta/erbB4 signaling. J Biol Chem 279: 51141-51147, 2004.

16. Ky B, Kimmel SE, Safa RN, Putt ME, Sweitzer NK, Fang JC, Sawyer DB and Cappola TP: Neuregulin-1 beta is associated with disease severity and adverse outcomes in chronic heart failure. Circulation 120: 310-317, 2009.
17. Geisberg CA, Wang G, Safa RN, Smith HM, Anderson B, Peng XY, Veerkamp B, Zhao DX, Blakemore D, Yu C and Sawyer DB: Circulating neuregulin-1 $\beta$ levels vary according to the angiographic severity of coronary artery disease and ischemia. Coron Artery Dis 22: 577-582, 2011.

18. Kalinowski A, Plowes NJ, Huang Q, Berdejo-Izquierdo C, Russell RR and Russell KS: Metalloproteinase dependent cleavage of neuregulin and autocrine stimulation of vascular endothelial cells. Faseb J 24: 2567-2575, 2010.

19. Lemmens K, Doggen K and De Keulenaer GW: Role of neuregulin-1/ErbB signaling in cardiovascular physiology and disease: Implications for therapy of heart failure. Circulation 116: 954-960, 2007

20. Reiss Y, Droste J, Heil M, Tribulova S, Schmidt MH, Schaper W, Dumont DJ and Plate KH: Angiopoietin-2 impairs revascularization after limb ischemia. Circ Res 101: 88-96, 2007.

21. Lok JL, Sardi SP, Guo S, Besancon E, Ha DM, Rosell A, Kim WJ, Corfas G and Lo EH: Neuregulin-1 signaling in brain endothelial cells. J Cereb Blood Flow Metab 29: 39-43, 2009.

22. Zhao YY, Sawyer DR, Baliga RR, Opel DJ, Han X, Marchionni MA and Kelly RA: Neuregulins promote survival and growth of cardiac myocytes. Persistence of erbb2 and erbb4 expression in neonatal and adult ventricular myocytes. J Biol Chem 273: 10261-10269, 1998.

23. Li B, Xiao J, Li Y, Zhang J and Zeng M: Gene transfer of human neuregulin-1 attenuates ventricular remodeling in diabetic cardiomyopathy rats. Exp Ther Med 6: 1105-1112, 2003. 